\title{
ON THE EXISTENCE OF BOUNDARY VALUES OF A CLASS OF BEPPO LEVI FUNCTIONS
}

\author{
BY \\ HANS WALLIN
}

1. Introduction. Let $f$ be a function which is defined and continuous in the interior $U$ of the unit-sphere in the $m$-dimensional Euclidean space $R^{m}$ and has partial derivatives of the first order a.e. in $U$ so that

$$
\int_{U}|\operatorname{grad} f(P)|^{2}(1-|P|)^{\alpha} d P<\infty, \quad 0 \leqq \alpha<1
$$

where $|P|$ is the distance from the origin to a point $P$ in $R^{m}$. Beurling $[1, \mathrm{p} .5]$ has proved that if $f$ is harmonic in $U$ and $m=2$ and $\alpha=0$ then $f$ has finite radial limits except when approaching a certain exceptional set on the boundary of $U$ having logarithmic capacity zero. After that Deny [5, p. 175] proved, for a general $m$ and $\alpha=0$, that if $f$ is a continuous Beppo Levi function then $f$ has finite radial limits except on an exceptional set having capacity zero with respect to the kernel $r^{-(m-2)}$. For $m=2$, Carleson [4, p. 48] proved a corresponding theorem for a general $\alpha$ and an exceptional set having capacity zero with respect to the kernel $r^{-\alpha}$. Using techniques from [6] and [10] and a method different from that of Carleson we shall generalize to higher dimensions his theorem with a general $\alpha$ and with $f$ absolutely continuous in the same way as a Beppo Levi function but for a half-space $R_{+}^{m}$ bounded by a hyperplane instead of for the sphere (Theorem 1). Let $P=(X, y)=\left(x_{1}, x_{2}, \cdots, x_{m-1}, y\right)$ be a point in $R_{+}^{m}$ and $y>0$ the distance from $P$ to the boundary of $R_{+}^{m}$ which we identify with the $(m-1)$-dimensional Euclidean space $R^{m-1}$ with points $X=\left(x_{1}, x_{2}, \cdots, x_{m-1}\right)$. Then the analogue of (1.1) is the condition

$$
\iint_{\Omega}|\operatorname{grad} f(X, y)|^{2} y^{\alpha} d X d y<\infty, \quad 0 \leqq \alpha<1,
$$

for every bounded domain $\Omega$ in $R_{+}^{m}$.

Using a familiar method we prove in Theorem 2 a converse of Theorem 1 showing that Theorem 1 is best possible as to the size of the exceptional set. If $f$ is harmonic, we can strengthen the result of Theorem 1 by proving the existence of nontangential limits (Theorem 3).

Received by the editors March 3, 1965. 
Let for $X_{0} \in R^{m-1}, V\left(X_{0}, \beta, h\right)$, where $\beta, h>0$, be the truncated cone

$$
\left\{(X, y)|| X-X_{0} \mid<\beta y, 0<y<h\right\}
$$

and consider the "generalized area integral"

$$
\iint_{V\left(X_{0}, \beta, h\right)} \frac{|\operatorname{grad} f(X, y)|^{2}}{y^{m-1-\alpha}} d X d y, \quad 0 \leqq \alpha \leqq 1 .
$$

Stein [9, Theorem 1] has proved that if $f$ is harmonic in $R_{+}^{m}$ and (1.3) is finite for $\alpha=1$ for every $X_{0}$ belonging to a given measurable set $E, E \subset R^{m-1}$ where $\beta$ and $h$ may depend on $X_{0}$-then $f$ has nontangential limits a.e. on $E$. There is a close connection between the integrals (1.2) and (1.3) (compare Spencer [8], Calderon [3] and Stein [9] for the case $\alpha=1$ ). This connection and the Theorems 1, 2 and 3 immediately give the following partial analogue of Stein's theorem when $\alpha<1$ (see Theorem 4): Let $0 \leqq \alpha<1$. If $f$ is defined and continuous in $R_{+}^{m}$ and absolutely continuous in the usual way and if, for some fixed $\beta$ and $h$, the integral (1.3) is a function of $X_{0}$ which is locally summable in $R^{m-1}$, then $f$ has finite normal limits except on a set having capacity zero with respect to the kernel $r^{-(m-2+\alpha)}$. Like Theorem 1 this is best possible as to the size of the exceptional set and if $f$ is harmonic we can assert the existence of nontangential limits.

In $\$ 7$ we briefly discuss generalizations of the above to the case when we in (1.2) replace $y^{\alpha}$ by a more general weight function $\{p(y)\}^{-1}$.

2. Preliminaries. Let $R_{+}^{m}, m \geqq 2$, denote the upper half-space of $R^{m}$. We identify $R_{m-1}$ with the (m-1)-dimensional hyperplane which is the boundary of $R_{+}^{m}$ in the Euclidean space $R^{m}$. We denote by a capital letter $X, X=\left(x_{1}, \cdots, x_{m-1}\right)$, a point in $R^{m-1}$ and by

$$
P=(X, y)=\left(x_{1}, x_{2}, \cdots, x_{m-1}, y\right), \quad X \in R^{m-1},-\infty<y<\infty
$$

a point in $R^{m}$. Thus $R_{+}^{m}$ is the set of points $P=(X, y), X \in R^{m-1}$, with $y>0 .|P|$ and $|X|$ denote distances in $R^{m}$ and $R^{m-1}$, respectively.

We write integrals over sets $E_{1} \subset R^{m}$ and $E_{2} \subset R^{m-1}$ as

$$
\int_{E_{1}}(\cdot) d P \text { or } \iint_{E_{1}}(\cdot) d X d y \text { and } \int_{E_{2}}(\cdot) d X .
$$

If no sets $E_{1}$ and $E_{2}$ are indicated, the integrals are taken over the whole spaces $R^{m}$ and $R^{m-1}$ respectively.

By an $m$-dimensional sphere we mean the set of points $P \in R^{m}$ satisfying $\left|P-P_{0}\right| \leqq r$ for some $P_{0} \in R^{m}$ and some $r>0 . V\left(X_{0}, \beta, h\right)$ is the truncated cone defined in the introduction.

$K$, the kernel, will be a function defined in the interval $r>0$ which is finite, 
continuous, nonincreasing and non-negative. If $\mu$ is a positive measure in $R^{m}$, i.e. a completely additive, positive set function, we define the $K$-potential of $\mu, u_{K}^{\mu}$, by

$$
u_{K}^{\mu}(Q)=\int K(|Q-P|) d \mu(P), \quad Q \in R^{m},
$$

and the energy integral $I_{K}(\mu)$ by

$$
I_{K}(\mu)=\iint K(|P-Q|) d \mu(P) d \mu(Q) .
$$

If $\mu$ is absolutely continuous with density $g, d \mu(x)=g(x) d(x)$, we also write $u_{K}^{g}$ instead of $u_{K}^{\mu}$. The support of a measure $\mu$ is denoted by $S_{\mu}$. The K-capacity of a Borel set $E, C_{K}(E)$, is defined as

$$
C_{K}(E)=\left\{\inf I_{K}(v)\right\}^{-1},
$$

where the infimum is taken over the class of all positive measures $v$ with $S_{v} \subset E$ and total mass $v(E)=1$. For $K(r)=r^{-\gamma}, 0<\gamma<m$, we also speak about the $\gamma$-capacity and use the notations $u_{\gamma}^{\mu}, I_{\gamma}(\mu)$ and $C_{\gamma}(E)$, respectively.

The $\gamma$-dimensional measure, $0<\gamma<m$, of a set $E, L_{\gamma}(E)$, is defined as

$$
\inf \sum_{v} r_{v}^{\gamma}
$$

where the infimum is taken over all denumerable coverings of $E$ by families of $m$-dimensional spheres with radii $\left\{\boldsymbol{r}_{v}\right\}$.

$C_{0}(E)$ is, as usual, to be interpreted as the logarithmic capacity, i.e. the capacity with respect to the kernel $-\log r$, and analogously for $L_{0}(E)$. This case, which corresponds to $m=2, \alpha=0$ in the Theorems $1-4$ below, sometimes requires simple modifications of the proofs, modifications which we omit.

In the proof of Theorem 1 we need the following lemma:

Lemma 1. Let $K$ be a kernel and $O$ an open set in $R^{m}$. Suppose that $g_{i}$, $i=1,2$, are functions having the property that, for every $\varepsilon>0$, there exists a Borel set $E, E \subset O$, with $C_{K}(E)<\varepsilon$ so that the restrictions of $g_{1}$ and $g_{2}$ to $O-E$ both are defined at every point of $O-E$ and continuous. Suppose also that $g_{1}(P)=g_{2}(P)$ for a.e. $P$ in $O$ and that the subset of $O$ where $g_{1}(P)=g_{2}(P)$ is a Borel set. Then $g_{1}(P)=g_{2}(P)$ on $O$ except on a subset of $K$-capacity zero.

When writing $g_{1}(P)=g_{2}(P)$ we of course assume that $g_{1}$ and $g_{2}$ are defined at $P$. This lemma was proved in $\left[10, \mathrm{p}\right.$. 72] for the case when $O=R^{m}$. The case with a general $O$ is proved analogously. Notice that there may exist a set of $K$-capacity zero where $g_{i}$ is not defined, $i=1,2$.

3. A general boundary value theorem. A function is said to be absolutely 
continuous on a line or a half-line if the restriction of the function to an arbitrary compact interval of the line or the half-line is absolutely continuous.

THEOREM 1. Let the real function $f$ be defined and continuous in $R_{+}^{m}$ and assume that $f$ is an absolutely continuous function of $x_{i}$ on almost every line in $R_{+}^{m}$ parallel to the $x_{i}$-axis for $i=1,2, \cdots, m-1$, and that $f$ is an absolutely continuous function of $y$ in $R_{+}^{m}$ for almost every $X$ in $R^{m-1}$. Let $0 \leqq \alpha<1$ and assume that $\left(^{1}\right)$

$$
\iint_{\Omega}|\operatorname{grad} f(X, y)|^{2} y^{\alpha} d X d y<\infty
$$

for every bounded domain $\Omega$ in $R_{+}^{m}$. Then $\lim _{y \rightarrow 0} f(X, y)$ exists and is finite for all $X$ in $R^{m-1}$ except when $X$ belongs to a certain Borel set $E$ with $C_{m-2+\alpha}(E)=0$.

REMARKS. It will appear from the proof that the condition that $f$ be continuous in $R_{+}^{m}$ may be changed to the condition that $f$ be continuous in $R_{+}^{m}$ except on a Borel set having $(m-2+\alpha)$-capacity zero. Furthermore, it is enough to make assumptions on $f$ in a set $\{(X, y) \mid 0<y<h\}$ where $h>0$ is a fixed constant.

Proof of Theorem 1. First we observe that it is enough to consider the case when $f$ has a compact support. This is a consequence of the fact that it suffices to prove that $f$ has boundary values in the way asserted inside every $m$-dimensional sphere $S$ with center in $R^{m-1}$ and that we may therefore replace $f$ by $f_{0}=f \cdot \phi$ where $\phi$ has a compact support, is infinitely differentiable and identically equal to 1 inside $S$. Then $f_{0}$ has a compact support and is absolutely continuous on every line where $f$ is absolutely continuous and, finally, (3.1) is satisfied with $f$ replaced by $f_{0}$ which is a consequence of the estimate

$$
\left|\operatorname{grad} f_{0}\right|^{2} \leqq \text { const. }\left(f^{2}+|\operatorname{grad} f|^{2}\right)
$$

and the fact that $f^{2}$ is locally summable in $R_{+}^{m}$. That $f^{2}$ is locally summable in $R_{+}^{m}$ is realized by representing $f(X, y)$ as the integral of its partial derivative with respect to $y$ almost everywhere in $R_{+}^{m}$ and using Schwarz's inequality on the integral to introduce the integral (3.1).

Hence we assume that $f$ has a compact support. We extend $f$ to all the points $(X, y)$ with $y<0$ by the definition

$$
f(X, y)=f(X,-y), \quad y<0 .
$$

Then we get the condition

$$
\iint_{R^{m}}|\operatorname{grad} f(X, y)|^{2}|y|^{\alpha} d X d y<\infty .
$$

(1) Note that the conditions on $f$ imply that the partial derivatives of the first order of $f$ exist a.e. in $R_{+}^{m}$ and are measurable. 
This and Schwarz's inequality give, as $\alpha<1$,

$$
\iint|\operatorname{grad} f(X, y)| d X d y<\infty .
$$

According to the assumptions $f$ is a.c. (absolutely continuous) on almost every line parallel to the $x_{i}$-axis, $i=1,2, \cdots, m-1$. But $f$ is also a.c. on a.e. line parallel to the $y$-axis which is realized in this way: $f$ is clearly a.c. on a line $l$ parallel to the $y$-axis if $f$ is a.c. on every compact subinterval of $l \cap R_{+}^{m}$ and $l$ is determined by points with a value of $X$ such that

$$
\int_{0}^{b}\left|\frac{\partial f(X, y)}{\partial y}\right| d y<\infty
$$

for a given $b>0$, which, according to (3.3), is true for a.e. $X$.

From (3.3) and the facts about the absolute continuity, we conclude [cf. 7, p. 315] that $f$, considered as a distribution, belongs to the class $B L_{1}\left(L_{\text {loc }}^{1}\right)$ of distributions having partial derivatives of the first order which are locally integrable functions.

As $f$ has a compact support and belongs to $B L_{1}\left(L_{\text {loc }}^{1}\right)$ we get the following representation formulas of $f$ [cf. for instance 10, p. 71], if we, to get more concise formulas, for a moment introduce a symmetrical notation by putting the $m$ th coordinate $y=x_{m}$, i.e. $P=(X, y)=\left(x_{1}, \cdots, x_{m-1}, x_{m}\right)$ and

$$
\begin{aligned}
P^{\prime} & =\left(X^{\prime}, y^{\prime}\right)=\left(x_{1}^{\prime}, \cdots, x_{m-1}^{\prime}, x_{m}^{\prime}\right), \\
f\left(P^{\prime}\right) & =M_{1} \sum_{i=1}^{m} \int \frac{\partial}{\partial x_{i}}\left|P^{\prime}-P\right|^{2-m} \frac{\partial}{\partial x_{i}} f(P) d P,
\end{aligned}
$$

a.e., if $m \geqq 3, M_{1}$ constant, and

$$
f\left(P^{\prime}\right)=M_{2} \sum_{i=1}^{2} \int \frac{\partial}{\partial x_{i}} \log \left|P^{\prime}-P\right| \frac{\partial}{\partial x_{i}} f(P) d P,
$$

a.e., if $m=2, M_{2}$ constant.

Let $v$ be the function which is defined and coincides with the right member of (3.4) [(3.5) if $m=2]$ at all the points where this right member is well-defined.

We need the following lemma:

LEMMA 2. Let $v$ be the function defined above. For every $\varepsilon>0$ there exists an open set $\Omega_{\varepsilon}$ with $C_{m-2+\alpha}\left(\Omega_{\varepsilon}\right)<\varepsilon$ such that the restriction of $v$ to the complement of $\Omega_{\varepsilon}$ is defined, finite and continuous everywhere in the complement of $\Omega_{\varepsilon}$.

By means of Lemma 2, which is proved below, it is now easy to complete the proof of Theorem 1. From Lemma 2 and Lemma 1 used with $O=R_{+}^{m}$ and $K(r)=r^{-(m-2+\alpha)}$ we obtain that the equality (3.4) $[(3.5)$ if $m=2]$ is true, not 
only a.e., but everywhere in $R_{+}^{m}$ except on a subset $N$ having $(m-2+\alpha)$ capacity zero. Now we use the fact that the $(m-2+\alpha)$-capacity of a Borel set in $R_{+}^{m}$ is larger than or equal to the $(m-2+a)$-capacity of its orthogonal projection on $R^{m-1}$. As pointed out by Brelot [2, p. 330] this follows easily when $\alpha=0$ from the fact that, for compact sets $F,\left\{C_{m-2}(F)\right\}^{-1}$ equals the transfinite diameter of $F$. However, even for a general kernel $K,\left\{C_{K}(F)\right\}^{-1}$ equals the transfinite diameter of $F$ which corresponds to the kernel $K$ and so Brelot's result follows also for more general kernels $K$, in particular for $K(r)=r^{-(m-2+\alpha)}$. This gives, if $\Omega_{\varepsilon}, \varepsilon>0$, is the set in Lemma 2 and $\left(\Omega_{\varepsilon} \cup N\right)_{1}$ is the orthogonal projection of $\Omega_{\varepsilon} \cup N$ on $R^{m-1}$,

$$
C_{m-2+\alpha}\left(\left(\Omega_{\varepsilon} \cup N\right)_{1}\right) \leqq C_{m-2+\alpha}\left(\Omega_{\varepsilon} \cup N\right)=C_{m-2+\alpha}\left(\Omega_{\varepsilon}\right)<\varepsilon .
$$

If $X_{0} \in R^{m-1}-\left(\Omega_{\varepsilon} \cup N\right)_{1}$ and $l_{0}$ is the line through $\left(X_{0}, 0\right)$ parallel to the $y$-axis, then the equality (3.4) [(3.5) if $m=2]$ is consequently valid everywhere on $l_{0} \cap R_{+}^{m}$ and the restriction of $v$ to $l_{0}$ is finite and continuous. Thus $\lim _{y \rightarrow 0} f\left(X_{0}, y\right)$ exists and is finite. As $\varepsilon$ can be chosen arbitrarily small this and (3.6) show that $f$ has finite normal limits except on a set having $(m-2+\alpha)$-capacity zero.

It remains to prove Lemma 2. To do this we need another lemma:

LEMMA 3. Let $g$ be a measurable, non-negative function defined in $R^{m}$ such that

$$
\iint|g(X, y)|^{2}|y|^{\alpha} d X d y<\infty, \quad 0 \leqq \alpha<1
$$

Let $G_{a}, a>0$, be the set of points $P^{\prime}$ where the potential

$$
u_{m-1}^{g}\left(P^{\prime}\right)=\int \frac{g(P)}{\left|P^{\prime}-P\right|^{m-1}} d P
$$

is larger than $a$. Then

$$
C_{m-2+\alpha}\left(G_{a}\right) \leqq \text { const } \because a^{-2} \iint|g(X, y)|^{2}|y|^{\alpha} d X d y,
$$

where the constant only depends on $\alpha$ and $m$.

Proof of Lemma 3. We shall prove that (3.8) is satisfied with $G_{a}$ replaced by a set $G_{a}^{\prime}$ where $G_{a}^{\prime}=G_{a} \cap\left(R_{+}^{m} \cup R^{m-1}\right)$. The fact that (3.8) is satisfied with $G_{a}$ replaced by $G_{a}-G_{a}^{\prime}$ also, is proved completely analogously by means of which the lemma then follows.

Let $\mu$ be a positive measure of total mass 1 supported by $G_{a}^{\prime}$. If $P=(X, y)$ and $A_{\alpha}(g)=\iint|g(X, y)|^{2}|y|^{\alpha} d X d y$, this gives by means of Schwarz's inequality, 


$$
\begin{aligned}
a^{2} & <\left(\int u_{m-1}^{g}\left(P^{\prime}\right) d \mu\left(P^{\prime}\right)\right)^{2} \leqq A_{\alpha}(g) \cdot \iint|y|^{-\alpha}\left\{u_{m-1}^{\mu}(P)\right\}^{2} d X d y \\
& =A_{\alpha}(g) \cdot \iint d \mu\left(P^{\prime}\right) d \mu\left(P^{\prime \prime}\right) \iint \frac{1}{|y| \alpha\left|P^{\prime}-P\right|^{m-1} \cdot\left|P^{\prime \prime}-P\right| m-1} d X d y .
\end{aligned}
$$

Since $P^{\prime}$ and $P^{\prime \prime}$ in the last integral can be considered as points in $R_{+}^{m} \cup R^{m-1}$, we realize by means of Lemma 4 which is stated below, that

$$
a^{2}<\text { const. } A_{\alpha}(g) \cdot I_{m-2+\alpha}(\mu),
$$

where the constant depends only on $\alpha$ and $m$. By taking the infimum over all $\mu$ we obtain that (3.8) is true with $G_{a}$ replaced by $G_{a}^{\prime}$ which gives the lemma.

Lemma 4. Let $P=(X, y), P^{\prime}, P^{\prime \prime} \in R_{+}^{m} \cup R^{m-1}$ and let $m+\alpha>2,0 \leqq \alpha<1$. Then

$$
\iint \frac{d X d y}{|y| \alpha\left|P^{\prime}-P\right|^{m-1} \cdot\left|P^{\prime \prime}-P\right|^{m-1}} \leqq \text { const. } \frac{1}{\left|P^{\prime}-P^{\prime \prime}\right|^{m-2+\alpha}},
$$

where the constant only depends on $\alpha$ and $m$.

Lemma 4 was proved in a little different form by Carleson $[4$, p. 50] for the case $m=2$. The general case can be proved analogously. See also the proof of Lemma $4^{\prime}$ in $\$ 7$.

Proof of Lemma 2. We shall prove Lemma 2 by a method which has been used by Deny [6, p. 369] to show a lemma of the same kind.

We first observe that Lemma 3 shows that the integrals in the formula (3.4) [(3.5) if $m=2]$ are absolutely convergent and consequently $v$ well-defined except on a set of $(m-2+\alpha)$-capacity zero. In fact, if we use Lemma 3 with $g$ defined by

$$
g(P)=\sum_{1}^{m}\left|\frac{\partial}{\partial x_{i}} f(P)\right|
$$

in the symmetric notation where $P=(X, y)=\left(x_{1}, \cdots, x_{m-1}, x_{m}\right)$, then (3.7) is satisfied due to (3.2) and so $u_{m-1}^{g}$ is finite except on a set of $(m-2+\alpha)$-capacity zero. But as a consequence of (3.9) a straightforward estimate shows that the integrals in (3.4) [(3.5) if $m=2]$ are absolutely convergent at those points where $u_{m-1}^{g}$ is finite.

Now let $f_{i n}, i=1,2, \cdots, m ; n=1,2, \cdots$, be continuous functions in $R^{m}$ with compact supports and put, in the symmetric notation where $y=x_{m}$,

$$
g_{n}(P)=\sum_{i=1}^{m}\left|f_{i n}(P)-\frac{\partial}{\partial x_{i}} f(P)\right|, \quad n=1,2, \cdots .
$$


We choose the functions $f_{\text {in }}$ such that

$$
\iint\left|g_{n}(P)\right|^{2}|y|^{\alpha} d X d y<4^{-n}, \quad P=(X, y), \quad n=1,2, \cdots
$$

It is easy to see that such a choice is possible as $f$ satisfies (3.2). We define the continuous functions $v_{n}$ in $R^{m}, n=1,2, \cdots$, by

$$
v_{n}\left(P^{\prime}\right)=M_{1} \sum_{i=1}^{m} \int \frac{\partial}{\partial x_{i}}\left|P^{\prime}-P\right|^{2-m} f_{i n}(P) d P
$$

for $m>2$ and analogously for $m=2$ [cf. the formulas (3.4) and (3.5)]. Disregarding the set of $(m-2+\alpha)$-capacity zero where $v$ is not well-defined we get

$$
\left|v\left(P^{\prime}\right)-v_{n}\left(P^{\prime}\right)\right| \leqq \text { const. } u_{m-1}^{g_{n}}\left(P^{\prime}\right)
$$

where $g_{n}$ is defined by the formula (3.10) and where the constant only depends on $m$ and on the constants $M_{1}$ and $M_{2}$ in (3.4) and (3.5). Let

$$
e_{n}=\left\{P^{\prime} \mid u_{m-1}^{g_{n}}\left(P^{\prime}\right)>2^{-n / 2}\right\}
$$

Due to (3.11) we then obtain from Lemma 3 used with $g=g_{n}$,

$$
C_{m-2+a}\left(e_{n}\right) \leqq \text { const. } \cdot 2^{-n} \text {. }
$$

If we put $E_{k}=\bigcup_{n=k}^{\infty} e_{n}, E_{k}$ is open and

$$
C_{m-2+\alpha}\left(E_{k}\right) \leqq \sum_{n=k}^{\infty} C_{m-2+\alpha}\left(e_{n}\right) \leqq \text { const. } \cdot 2^{-k},
$$

i.e. $C_{m-2+a}\left(E_{k}\right) \rightarrow 0$ when $k \rightarrow \infty$. But $v_{n}$ tends to $v$ uniformly on the complement of $E_{k}$ for every $k=1,2, \cdots$, and so the restriction of $v$ to the complement of $E_{k}$ is continuous for every $k$, which proves Lemma 2.

The proof of Theorem 1 is thus complete.

4. A converse of Theorem 1. Theorem 1 is best possible in the following sense:

THEOREM 2. Let $F$ be a compact set in $R^{m-1}$ with

$$
C_{m-2+\alpha}(F)=0, \quad \text { where } 0 \leqq \alpha<1 .
$$

Then there exists a function $f$ defined and infinitely differentiable in $R_{+}^{m}$ satisfying

$$
\iint_{R_{+}^{m}}|\operatorname{grad} f(X, y)|^{2} y^{\alpha} d X d y<\infty
$$

and

$$
f(X, y) \rightarrow+\infty \text { when }(X, y) \rightarrow\left(X_{0}, 0\right), \quad y>0, X_{0} \in F
$$


Proof. The method which we shall use is well known (cf. Carleson [4, p. 47]). Let $\beta=m-2+\alpha$. For every $\varepsilon>0$ there exists a finite union $E$ of closed $(m-1)$-dimensional spheres in $R^{m-1}$ so that $E \supset F$ and $C_{\beta}(E)=\varepsilon$. Let $y$ be the equilibrium distribution of total mass $\varepsilon$ belonging to $E$ and the kernel $r^{-\beta}$, i.e. the positive measure with total mass $\varepsilon, S_{v} \subset E$ and

$$
I_{\beta}(v)=\inf _{\tau} I_{\beta}(\tau)
$$

where the infimum is taken over all positive measures $\tau$ with $S_{\tau} \subset E$ and $\tau(E)=\varepsilon$. We have $I_{\beta}(v)=\varepsilon$ and, due to the regularity of $E$,

$$
u_{\beta}^{v}(P)=1 \text { if } P \in E .
$$

As $u_{\beta}^{v}(P)$ is identically equal to 1 on $S_{v}, u_{\beta}^{v}$ is continuous everywhere. In particular we have

$$
\lim _{P \rightarrow\left(X_{0}, 0\right)} u_{\beta}^{v}(P)=1 \text { if } X_{0} \in E .
$$

Now we consider a sequence of compact sets $\left\{F_{n}\right\}$ where each $F_{n} \in R^{m-1}$ is a finite union of $(m-1)$-dimensional spheres so that

$$
F_{n} \supset F_{n+1} \supset F \text { and } C_{\beta}\left(F_{n}\right)<2^{-n} \text {, all } n \text {. }
$$

Let $\mu_{n}$ be the equilibrium distribution of total mass $C_{\beta}\left(F_{n}\right)$ belonging to $F_{n}$ and $r^{-\beta}$ and put $\mu=\Sigma_{1}^{\infty} \mu_{i}$ (weak convergence). We define $f$ by

$$
f(P)=u_{\beta}^{\mu}(P), \quad P \in R_{+}^{m} .
$$

Then $f$ is infinitely differentiable in $R_{+}^{m}$ and

$$
\liminf f(P) \geqq \liminf \sum_{1}^{N} u_{\beta}^{\mu_{n}}(P)=N, \text { all } N, \text { when } P \rightarrow\left(X_{0}, 0\right) \in F,
$$

which shows that (4.2) is satisfied. Furthermore, we have, if $P=(X, y) \in R_{+}^{m}$,

$$
\left|\frac{\partial}{\partial x_{i}} f(X, y)\right| \leqq \text { const. } \int \frac{d \mu\left(P^{\prime}\right)}{\left|P-P^{\prime}\right|^{m-1+\alpha}}, \quad i=1,2, \cdots, m-1,
$$

and analogously for $|(\partial / \partial y) f(P)|$. Hence the integral (4.1) is majorized by a constant times

$$
\iint d \mu\left(P^{\prime}\right) d \mu\left(P^{\prime \prime}\right) \iint_{R_{+}^{m}} \frac{y^{\alpha}}{\left|P-P^{\prime}\right|^{m-1+\alpha} \cdot\left|P-P^{\prime \prime}\right|^{m-1+\alpha}} d X d y
$$

and as for instance $P^{\prime} \in R^{m-1}$ we have $y^{\alpha} \leqq\left|P-P^{\prime}\right|^{\alpha}$ which clearly gives that the integral (4.1) is majorized by a constant times $I_{\beta}(\mu)$. But $I_{\beta}(\mu)$ is finite because 


$$
I_{\beta}(\mu)<2 \sum_{j=1}^{\infty} \sum_{i=1}^{j} \int u_{\beta}^{\mu_{i}}(P) d \mu_{j}(P) \leqq \text { const. } \sum_{j=1}^{\infty} \sum_{i=1}^{j} \mu_{j}\left(F_{j}\right)
$$

which is finite as

$$
\mu_{j}\left(F_{j}\right)=C_{\beta}\left(F_{j}\right)<2^{-j} .
$$

Hence also (4.1) is satisfied which proves Theorem 2.

5. The case when $f$ is harmonic. Theorem 1 asserts the existence of boundary values of $f$, except on a certain set, when approaching a point in $R^{m-1}$ along the normal. Clearly the conclusion of limits remains valid also if we approach $R^{m-1}$ along half-lines in $R_{+}^{m}$ forming an arbitrarily chosen, fixed angle with the hyperplane $R^{m-1}$. We may also approach a given point in $R^{m-1}$ along lines with different directions and obtain an exceptional set of directions for which there exist no finite limits. However, for general functions, we cannot assert the existence of nontangential limits (cf. Carleson [4, p. 54]). On the other hand we shall prove that $f$ has nontangential limits if $f$ is harmonic in $R_{+}^{m}$.

The function $f$ defined in $R_{+}^{m}$ is said to have a nontangential limit $A$ at a point $X_{0} \in R^{m-1}$ if for every $\beta>0$,

$$
f(X, y) \rightarrow A \text { when } y \rightarrow 0 \text { and }(X, y) \in V\left(X_{0}, \beta, h\right) .
$$

THEOREM 3. Let $0 \leqq \alpha<1$. Suppose that the function $u$ is harmonic in $R_{+}^{m}$ and that

$$
\iint_{\Omega}|\operatorname{grad} u(X, y)|^{2} y^{\alpha} d X d y<\infty
$$

for all bounded domains $\Omega$ in $R_{+}^{m}$. Then $u$ has finite nontangential limits for $X_{0} \in R^{m-1}$ except when $X_{0}$ belongs to a certain Borel set $E$ satisfying $C_{m-2+a}(E)=0$.

For the proof we need two lemmas.

Lemma 5. Let $g$ be a non-negative measurable function defined in $R_{+}^{m}$ such that

$$
\iint_{\Omega} g(X, y) d X d y<\infty
$$

for every bounded domain $\Omega$ in $R_{+}^{m}$. Let $0<\gamma \leqq m-1$ and let $\beta$ and $h$ be two fixed positive numbers. Then

$$
\iint_{V\left(X_{0}, \beta, h\right)} \frac{g(X, y)}{y^{\gamma}} d X d y<\infty
$$

except when $X_{0}$ belongs to a certain Borel set $E$ in $R^{m-1}$ with $L_{\gamma}(E)=0$ 
Proof. Suppose that the set $E$ where the integral (5.3) is infinite satisfies

$$
L_{\gamma}(E)>0 \text {. }
$$

According to a well-known result (cf. $[4$, p. 9]) there exists a compact subset $F$ of $E$ such that $L_{\gamma}(F)>0$. Furthermore, there exists, according to a result by Frostman (cf. $\left[4\right.$, p. 6]) a non-negative measure $\mu$ with total mass $1, S_{\mu} \subset F$ and

$$
\mu(S) \leqq \text { const. } r^{\gamma}
$$

for every $m$-dimensional sphere $S$ with radius $r$. We put $V\left(X_{0}\right)=V\left(X_{0}, \beta, h\right)$ and let $\psi\left(X_{0} ; X, y\right)$ denote the indicator of $V\left(X_{0}\right)$, i.e. $\psi\left(X_{0} ; X, y\right)=1$ if $(X, y) \in V\left(X_{0}\right)$ and $\psi\left(X_{0} ; X, y\right)=0$ elsewhere. Then we get, if $\Omega=\bigcup_{x_{0} \in F} V\left(X_{0}\right)$,

$$
\begin{aligned}
\infty & =\iint_{F}\left\{\int_{V\left(X_{0}\right)} g(X, y) \cdot y^{-\gamma} d X d y\right\} d \mu\left(X_{0}\right) \\
& =\iint_{\Omega}\left\{\int_{F} \psi\left(X_{0} ; X, y\right) d \mu\left(X_{0}\right)\right\} g(X, y) \cdot y^{-\gamma} d X d y \\
& =\iint_{\Omega}\left\{\int_{\left|X-X_{0}\right|<\beta y} d \mu\left(X_{0}\right)\right\} g(X, y) \cdot y^{-\gamma} d X d y .
\end{aligned}
$$

But using (5.4) we see that the last integral is majorized by a constant times the convergent integral (5.2) which gives a contradiction and therefore we have $L_{\gamma}(E)=0$.

LEMMA 6. Let $\beta^{\prime}>\beta>0, h^{\prime}>h>0$ and let $q$ be a function defined in the interval $r>0$ which is non-negative, nonincreasing and satisfies

$$
q(r) \leqq A q(2 r), r>0,
$$

for some constant $A$. Suppose that $u$ is a harmonic function in $V\left(X_{0}, \beta^{\prime}, h^{\prime}\right)$ such that

$$
\iint_{V\left(X_{0}, \beta^{\prime}, h^{\prime}\right)}|\operatorname{grad} u(X, y)|^{2} \cdot q(y) d X d y<\infty .
$$

Then

$$
y^{m / 2}\{q(y)\}^{1 / 2}|\operatorname{grad} u(X, y)| \leqq M<\infty \text { for }(X, y) \in V\left(X_{0}, \beta, h\right),
$$

where $M$ is a constant only depending on $m, \beta^{\prime}, \beta, h^{\prime}, h, A$ and the value of the integral (5.5).

This lemma is proved in $[9$, p. 146] for a special choice of $q$. The proof is completely analogous in the general case. 
Proof of Theorem 3. In order to avoid some technical complications we shall prove the existence of nontangential limits inside an arbitrarily chosen $m$-dimensional sphere $S$ having center at the origin and we consider, instead of $u$, the function $u_{0}=\phi \cdot u$ where $\phi$ is infinitely differentiable, has a compact support and is identically equal to 1 inside $S$. Then (5.1) is satisfied with $u$ replaced by $u_{0}$ and $\Omega$ by $R_{+}^{m}$. Hence there exists a non-negative, nonincreasing function $q_{1}$ defined in the interval $r>0$ such that

$$
q_{1}(r) \leqq A q_{1}(2 r), r>0, \text { for some constant } A, q_{1}(r) \rightarrow \infty \text { when } r \rightarrow 0,
$$

and, finally,

$$
\iint_{R_{+}^{m}}\left|\operatorname{grad} u_{0}(X, y)\right|^{2} q_{1}(y) y^{\alpha} d X d y<\infty .
$$

Let $\beta$ and $h$ be two arbitrarily chosen positive numbers. It is enough to prove the existence of limits of $u_{0}$ inside cones $V\left(X_{0}, \beta, h\right)$. We choose $\beta^{\prime}>\beta$ and $h^{\prime}>h$. Lemma 5 used with

$$
g(X, y)=q_{1}(y)\left|\operatorname{grad} u_{0}(X, y)\right|^{2} y^{\alpha}
$$

gives, due to (5.6)

$$
\int_{V\left(X_{0}, \beta^{\prime}, h^{\prime}\right)} \frac{q_{1}(y)\left|\operatorname{grad} u_{0}(X, y)\right|^{2}}{y^{m-2}} d X d y<\infty
$$

except on a $X_{0}$-set having ( $\left.m-2+\alpha\right)$-dimensional measure zero and thus, in particular, except on a set of $(m-2+\alpha)$-capacity zero. From this fact and Theorem 1 we conclude that it is enough to prove that $u_{0}$ has a finite limit when we approach a point $X_{0}$, inside $V\left(X_{0}, \beta, h\right)$, where $X_{0}$ is a point in the interior of $S$ such that, firstly, the integral (5.7) is finite and, secondly, $u_{0}$ has a finite limit when approaching $X_{0}$ along the normal at $X_{0}$ of the hyperplane $R^{m-1}$. Let $X_{0}$ be such a point. According to the triangle inequality it suffices to show that

$$
u_{0}(X, y)-u_{0}\left(X_{0}, y\right) \rightarrow 0 \text { when } y \rightarrow 0 \text { and }(X, y) \in V\left(X_{0}, \beta, h\right) .
$$

But if $l$ is the line segment between $(X, y)$ and $\left(X_{0}, y\right)$ we have

$$
\left|u_{0}(X, y)-u_{0}\left(X_{0}, y\right)\right| \leqq\left|X-X_{0}\right| \sup _{l}\left|\operatorname{grad} u_{0}\left(X^{\prime}, y\right)\right|,
$$

and if we use (5.7) and Lemma 6 with

$$
q(r)=q_{1}(r) \cdot r^{-(m-2)}
$$

we get

$$
y\left\{q_{1}(y)\right\}^{1 / 2} \cdot\left|\operatorname{grad} u_{0}(X, y)\right| \leqq M<\infty,(X, y) \in V\left(X_{0}, \beta, h\right),
$$

which proves that (5.8) is majorized by 


$$
M \cdot \frac{\left|X-X_{0}\right|}{y} \cdot\left\{q_{1}(y)\right\}^{-1 / 2}
$$

which tends to zero when $y$ tends to zero and $(X, y) \in V\left(X_{0}, \beta, h\right)$. This proves Theorem 3.

6. Connection with the generalized area integral. The connection between the integral (3.1) and the "generalized area integral"

$$
A_{\alpha}\left(X_{0}\right)=\iint_{V\left(X_{0}, \beta, h\right)} \frac{|\operatorname{grad} f(X, y)|^{2}}{y^{m-1-\alpha}} d X d y
$$

leads to the following equivalent formulation of the Theorems 1, 2 and 3:

THEOREM 4. Let $0 \leqq \alpha<1, \beta>0$ and $h>0$ be fixed numbers.

(a) Suppose that $f$ is defined and continuous in $R_{+}^{m}$ and absolutely continuous in the same way as in Theorem 1 and that

$$
\int_{D} A_{\alpha}\left(X_{0}\right) d X_{0}<\infty
$$

for every bounded domain $D$ in $R^{m-1}$. Then $\lim _{y \rightarrow 0} f(X, y)$ exists and is finite except when $X$ belongs to a Borel set $E$ with $C_{m-2+\alpha}(E)=0$.

(b) If $F \subset R^{m-1}$ is compact and $C_{m-2+\alpha}(F)=0$, then there exists an infinitely differentiable function $f$ in $R_{+}^{m}$ satisfying (6.1) and

$$
f(P) \rightarrow+\infty, \quad P \rightarrow(X, 0), \quad P \in R_{+}^{m}, \quad X \in F .
$$

(c) If $u$ is harmonic in $R_{+}^{m}$ and satisfies (6.1), then $u$ has nontangential limits except on a Borel set having $(m-2+\alpha)$-capacity zero.

Theorem 4 is an immediate consequence of the Theorems 1, 2 and 3 (compare the remarks following after Theorem 1) and the following lemma:

LeMMA 7. Suppose we are given a real number $\alpha, \beta>0, h>0$ and a function $f$ defined and measurable in $R_{+}^{m}$. Then

$$
\int_{D} A_{\alpha}\left(X_{0}\right) d X_{0}<\infty
$$

for all bounded domains $D$ in $R^{m-1}$ if and only if

$$
\iint_{\Omega}|\operatorname{grad} f(X, y)|^{2} y^{\alpha} d X d y<\infty
$$

for all bounded domains $\Omega$ with $\Omega \subset\{(X, y) \mid 0<y<h\}$.

This lemma was essentially proved by Stein [9, p. 148 and p. 151] for the case $\alpha=1$ without being stated explicitly. The same method of proof is applicable for a general $\alpha$. 
7. A general weight function. The above theorems can be generalized by introducing a more general weight function than $y^{\alpha}$ in (3.1). We shall be content to state generalizations of the Theorems 1 and 2 .

Let $p$ be a strictly positive, finite, nonincreasing function defined for $r>0$ such that, for some constant $a<2$

$$
p(r) \leqq a p(2 r), \quad r>0 .
$$

We define the kernel $K$ by

$$
K(r)=\left\{\begin{array}{l}
\frac{p(r)}{r^{m-2}} \quad \text { if } m \geqq 3, \\
\int_{r}^{\infty} \frac{p(t)}{t} d t \quad \text { if } m=2,
\end{array}\right.
$$

where, for $m=2$, the integral is supposed to be finite if $r>0$. Then we have the following generalization of Theorem 1 :

THEOREM 1'. Suppose that $f$ is defined and continuous in $R_{+}^{m}$ and absolutely continuous in the same way as in Theorem 1 and that

$$
\iint_{\Omega}|\operatorname{grad} f(X, y)|^{2}\{p(y)\}^{-1} d X d y<\infty
$$

for all bounded domains $\Omega$ in $R_{+}^{m}$. Then $\lim _{y \rightarrow 0} f(X, y)$ exists and is finite except when $X$ belongs to a certain Borel set $E$ with $C_{K}(E)=0$.

If, furthermore, we assume that $p$ is infinitely differentiable in the interval $r>0$ and that the derivative $p^{\prime}$ is nondecreasing if $m \geqq 3$ we get the following generalization of Theorem 2:

THEOREM $2^{\prime}$. Let $F$ be a compact set in $R^{m-1}$ with $C_{K}(F)=0$. Then there exists a function $f$, defined and infinitely differentiable in $R_{+}^{m}$, such that the integral (7.2) is finite for $\Omega=R_{+}^{m}$ and

$$
f(P) \rightarrow+\infty, \quad P \rightarrow(X, 0), \quad P \in R_{+}^{m}, \quad X \in F .
$$

The condition (7.1) with $a<2$ guarantees that

$$
\int_{0}^{1} p(r) d r<\infty
$$

and, due to this fact, the Theorems $1^{\prime}$ and $2^{\prime}$ may be proved analogously to the Theorems 1 and 2 essentially by throughout changing $y^{\alpha}$ to $\{p(y)\}^{-1}$ and the kernel $r^{-(m-2+\alpha)}$ to the kernel $K(r)$. A new complication in the proof of Theorem $1^{\prime}$ is the proof of the following version of Lemma 4 which is needed to prove the new version of Lemma 3: 
Lemma $4^{\prime}$. Let $P=(X, y)$ and let $P^{\prime}$ and $P^{\prime \prime} \in R_{+}^{m} \cup R^{m-1}$. Then

$$
\iint \frac{p(|y|)}{\left|P^{\prime}-P\right|^{m-1} \cdot\left|P^{\prime \prime}-P\right|^{m-1}} d X d y \leqq \text { const. } K\left(\left|P^{\prime}-P^{\prime \prime}\right|\right),
$$

where the constant only depends on $m$ and the number $a, a<2$, occurring in (7.1).

Proof of Lemma 4'. See Carleson $[4$, p. 50] for the case $m=2$ and $p(r)=r^{-\alpha}$. The function $p$ causes technical complications in the estimations similar to those in the estimations on p. 76 in [10]. For that reason we only sketch the proof of the lemma.

As $p$ is nonincreasing simple considerations show that we may assume that one of the points $P^{\prime}$ and $P^{\prime \prime}$, for instance $P^{\prime}$, is situated in the hyperplane $R^{m-1}$ and that we then, by introducing $P-P^{\prime}$ as the new variable of integration, may reduce the proof of (7.3) to the proof of

$$
\int p(|y|) d y \int \frac{d X}{|P|^{m-1}\left|P-P^{\prime}\right| m-1} \leqq \text { const. } K\left(\left|P^{\prime}\right|\right),
$$

where $P=(X, y), P^{\prime} \in R_{+}^{m} \cup R^{m-1}$. (7.4) is proved by dividing the integration with respect to $y$ into two parts

(a) $|y|<2\left|P^{\prime}\right|$ and

(b) $|y| \geqq 2\left|P^{\prime}\right|$.

In the case (a) we make the following division of the integration, $P=(X, y)$,

(a1) $\left\{P|| P|\leqq| P^{\prime} \mid / 2\right\}$,

(a2) $\left\{P|| P-P^{\prime}|\leqq| P^{\prime} \mid / 2\right\}$,

(a3) $\left\{P|P|>\left|P^{\prime}\right| / 2,\left|P-P^{\prime}\right|>\left|P^{\prime}\right| / 2,|X|<2\left|P^{\prime}\right|\right\}$,

(a4) $\left\{P|| X|\geqq 2| P^{\prime} \mid\right\}$,

and in the case (b),

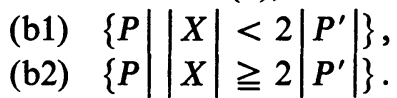

(7.4) is now proved by a systematic use of the facts that $p$ is nonincreasing and satisfies (7.1) with $a<2$. This completes our proof of Lemma $4^{\prime}$.

In the proof of Theorem $2^{\prime}$ we get

$$
\left|K^{\prime}(r)\right| \leqq \text { const. } \frac{K(r)}{r}, \quad r>0, \text { if } m \geqq 3,
$$

from the assumption on the derivative $p^{\prime}$. This is used in the estimation of the integral (7.2) for $f=u_{K}^{\mu}$ with a certain measure $\mu$. When $m=2$ we use the relation

$$
K^{\prime}(r)=-\frac{p(r)}{r}
$$


We also observe that in the proof of Theorem $2^{\prime}$ the analogue of the measure $\mu_{n}$ used in the proof of Theorem 2 is in general not an equilibrium distribution but a so called capacitary distribution of total mass $C_{K}\left(F_{n}\right)$ belonging to $F_{n}$ and the kernel $K$. Using known properties (cf. for instance [10, p. 57]) of the $K$-potential of this capacitary distribution it is easy to complete the pronf of Theorem 2'.

\title{
REFERENCES
}

1. A. Beurling, Ensembles exceptionnels, Acta Math. 72 (1940), 1-13.

2. M. Brelot, Points irréguliers et transformations continues en théorie du potentiel, J. Math. Pures Appl. 19 (1940), 319-337.

3. A. P. Calderon, On the theorem of Marcinkiewicz and Zygmund, Trans. Amer. Math. Soc. 68 (1950), 55-61. $1-81$.

4. L. Carleson, Selected problems on exceptional sets (mimeographed), Uppsala, 1961; pp.

5. J. Deny, Les potentiels d'énergie finie, Acta Math. 82 (1950), 107-183.

6. _- Sur la convergence de certaines intégrales de la théorie du potentiel, Arch. Math. 5 (1954), 367-370.

7. J. Deny and J. L. Lions, Les éspaces du type de Beppo Levi, Ann. Inst. Fourier (Grenoble) 5 (1955), 305-370.

8. D. C. Spencer, A function theoretic identity, Amer. J. Math. 65 (1943), 147-160.

9. E. M. Stein, On the theory of harmonic functions of several variables. II, Acta Math. 106 (1961), 137-174.

10. H. Wallin, Continuous functions and potential theory, Ark. Mat. 5 (1963), 55-84.

\author{
INSTITUTE FOR AdVANCEd Study, \\ Princeton, New Jersey \\ UNIVERSITY OF UPPSALA, \\ UpPSALA, Sweden
}

